\title{
Über die Darstellung von Glycocoll und über einige seiner Derivate
}

von

J. Mauthner und W. Suida.

Aus dem Laboratorium für angewandte medicinische Chemie an der k. k. Universität in Wien.

(Vorgelegt in der Sitzung am 17. Juli 1890.)

Bei der Fortsetzung früherer Untersuchungen, welche den in der Überschrift genannten Gegenstand betreffen, wurden einige Beobachtungen gewonnen, deren Mittheilung den Inhalt der folgenden Notizen bilden soll.

\section{Darstellung von Glycocoll.}

Am. Sehlusse anserer vor zwei Jahren veröffentlichten Mittheilung über die Gewinnung des Glycocolls aus Chloressigsäure ${ }^{1}$ erwähnten wir die Beobachtung, dass eine bedeutend bessere Ansbente an Glycocoll, als wir sie bei der Anwendung von kohlensaurem Natrium und wässerigem Ammoniak erzielt hatten, dadurch erreicht werde, dass man das kohlensature Natrium durch Bleicarbonat ersetzt. Bei der. Verfolgung dieser Beobachtung liessen wir auch wiederholt concentrirtes wässeriges Ammoniak in grossem Überschusse auf Chloressigsäure allein einwirken, ohne Bleicarbonat hinzuzufügen. Auch bei diesen Versuchen ergab sich eine nennenswerthe Ausbeute an Glycocoll und es zeigte sich, dass die wesentliche Bedingung furr eine solche in der Anwesenheit grosser Mengen überschüssigen Ammoniaks bei gewöhnlicher Temperatur ist. Die Ausbeuten an Glycocoll, welche man so erzielen kann, bleiben noch immer weit hinter der theoretischen Menge zuriick, sie übertreffen jedoch nicht

1 Monatshefte fiir Chemie, IX, 735. 
allein die von uns seinerzeit unter Anwendung von kohlensaurem Natrium erzielten Mengen, sondern auch jene, welche Nencki ${ }^{1}$ durch Erhitzen von Chloressigsäure mit trockenem kohlensauren Ammon erreicht hat.

Wenn seither auch von S. Gabriel und K. Kroseberg. ${ }^{2}$ eine Methode angegeben wurde, welche es gestattet, aus Chloressigsäure nahezu die theoretische Menge an salzsaurem Glycocoll zu gewinnen, so halten wir es doch nicht für überflüssig, unsere Erfahrungen mitzutheilen, da sich der von uns eingehaltene Vorgang sehr einfach gestaltet. Wir verfuhren in folgender Weise: Je $100 \mathrm{~g}$ Chloressigsäure wurden in circa $100 \mathrm{~cm}^{3}$ Wasser oder Alkohol gelöst und in 1 Liter concentrirter (20 bis $22 \%$ ) wässeriger Ammoniakflüssigkeit unter Abkühlen in kleinen Antheilen eingetragen. Nachdem die Flüssigkeit eine Woche bei Zimmertemperatur gestanden war', wurde sie eingedampft, bis das freie Ammoniak entwichen war und hierauf mit Bleioxyd bis nahe zum Verschwinden des Ammoniakgeruches gekocht. Dann wurde heiss filtrirt, das Ungelöste mit heissem Wasser ausgezogen, die erkalteten Filtrate neuerdings filtrirt und mit frischem Schwefelammonium rom Blei befreit. Die rom Schwefelblei getrennte Lösung wurde nahe zur Trockene eingedampft, der krystallinische Riickstand mit wenig Wasser anfgenommen und mit käuflichem Kupfercarbonat gekocht; beim Abkühlen erstarrte die heiss filtrirte Flüssigkeit zu einem Brei des nadelförmigen Glycocollkupfers. Die Krystalle wurden abgesangt und aus wenig Wasser unkrystallisirt. Unter bestimmten Umständen beobachteten wir bei diesem Vorgange das Auftreten blauvioletter, perlmutterglänzender Blätter, die sich dem Ansehen und Verhalten nach von den hellblauen Nadeln des bekannten Glycocollkupfers wesentlich unterschieden. Wir werden auf diese Beobachtung noch zurückkommen.

Die erste Mutterlange von dem rohen Glycocollkupfer lieferte beim freiwilligen Verdunsten noch erhebliche Mengen unreinen Kupfersalzes, das ebenfalls durch Umkrystallisiren aus wenig Wasser gereinigt wurde. Die Ausbeute an Glycocollkupfer, dessen

1 Berichte, XVI, 2827.

2 Berichte, XXII, 426. 
Reinheit durch Analyse jeweilen cotrolirt wurde, ${ }^{1}$ betrug im Durchschnitt $28 \cdot 5 \%$ mit der Minimalzahl von $23 \cdot 9 \%$. Wenn es sich darum handelt, das Kupfersalz zur Gewinnung von Glycocoll zu zerlegen, so empfiehlt es sich, dazu nicht Schwefelwasserstoff anzuwenden, da die Operation durch das Gelöstbleiben des Schwefelkupfers zu einer sehr unerquicklichen wird, sondern sich des farblosen Schwefelammoniums zu bedienen und nach Entfernung des Schwefelkupfers die Fliussigkeit einzadampfen. Die Entfernung der geringen Mengen von Schwefel, welche dem zurïckbleibenden Glycocoll beigemengt erscheinen, gelingt ohne jede Schwierigkeit durch Umkrystallisiren. Das Glycocoll wird nach diesem Vorgang quantitativ aus dem Kupfersalz gewonnen.

Ein so dargestelltes, aus der wässerigen Lösung durch Alkohol gefälltes Präparat gab bei der Analyse 32.01\% (berechnet: $32 \cdot 00$ ) Kohlenstoff und $6 \cdot 77 \%$ (berechnet: $6 \cdot 66$ ) Wasserstoff.

\section{Blätteriges Kupfersalz des Glycocolls.}

Wir haben friiher der Beobachtung Erwähnung gethan, dass beim Umkrystallisiren des Glycocollkupfers gelegentlich blauviolette, perlmutterglänzende Blättchen auftreten. Wir hielten dieselben anfangs fuir eine dem Glycocollkupfer beigemengte fremde Substanz, bis bei dem Versuche, eine grössere Menge dieser Blättchen nenerdings umzukrystallisiren, aus der heissen Lösung wieder die bekannten blauen Nadeln des gew öhnlichen Glycocollkupfers herausfielen und wir uns hiedurch und später durch die Analysen ïberzeugten, dass eine neue Form des Glycocollkupfers in den Blättchen vorliege. Wie wir wiederholt beobachten konnten, entsteht dieses blätterige Salz aus dem nadelförmigen dann, wenn zum Umkrystallisiren des Letzteren unzureichende Mengen von Wasser verwendet werden, wobei also eine gesättigte Lösung von Glycocollkupfer mit noch ungelöstem Glycocollkupfer erhitzt wird. Scheinbar geht das nicht gelöste Salz in die neue Form allmälig über. Durch rasches $\mathrm{Ab}$ filtriren und Auswaschen mit Wasser gelang es unschwer, grössere

1. Wir halten es für ïberflïssig, die zahlreichen Analysen, welche dabei ausgefiihrt wurden, einzeln anzuführen. 
Mengen dieses schwerer löslichen Kupfersalzes frei von dem nadelförmigen Glycocollkupfer in solcher Reinheit zu erlangen, dass es der Analyse unterworfen werden konnte. Dabei ergab sich, dass es genau dieselbe Zusammensetzung besitzt, wie das nadelförmige Salz. Die Analysen ergaben folgende Werthe:

I $0.5269 \mathrm{~g}$ des lufttrockenen Salzes verloren den grössten Theil ihres Wassers schon beim Stehen über Schwefelsäure, den Rest bei $110^{\circ}$ C., im Ganzen $0.0423 \mathrm{~g}$.

II $0.5193 \mathrm{~g}$ verloren bei $100^{\circ}$ C. $0.0411 \mathrm{~g}$ Wasser.

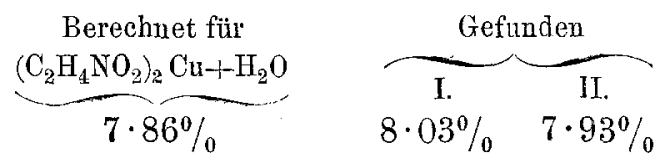

I. $0.3189 \mathrm{~g}$ bei $100^{\circ}$ getrocknet, gaben bei der Verbrennung $0 \cdot 1097 \mathrm{~g}$ Wasser $0 \cdot 2678 \mathrm{~g}$ Kohlensäure und $0 \cdot 1212 \mathrm{~g}$ Kupferoxyd.

II. $0 \cdot 4815 g$ bei $110^{\circ}$ getrocknet, gaben $0.1801 g$ Kupferoxyd. III. $0 \cdot 4782 g$ bei $100^{\circ}$ getrocknet, gaben $0 \cdot 1789 g$ Kupferoxyd. IV. $0.4872 g$ bei $105-110^{\circ}$ getrocknet, gaben $0.1827 g$ Kupferoxyd.

V. $0 \cdot 4224 \mathrm{~g}$ bei $110^{\circ}$ getrocknet, gaben $50.05 \mathrm{~cm}^{3}$ Stickstoff bei $16 \cdot 8^{\circ} \mathrm{C}$. und $737.5 \mathrm{~mm}$ Barometerstand.

Berechnet für

$\left(\mathrm{C}_{2} \mathrm{H}_{4} \mathrm{NO}_{2}\right)_{2} \mathrm{Cu}$

C. ...22.72\%

$\mathrm{H} \ldots 3 \cdot 79$

$\mathrm{N} \ldots .13 \cdot 25$

Cu...29:92

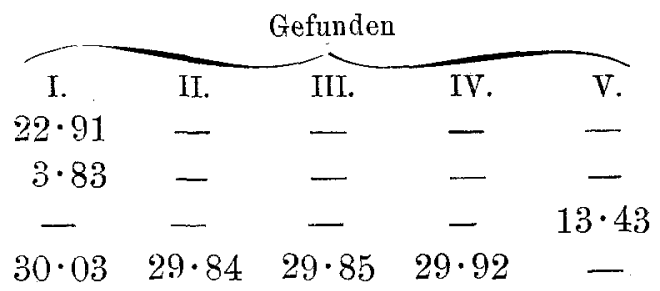

Schon gelegentlich dieser Analysen zeigte es sich, dass das Krystallwasser, welches in dem blätterförmigen Salze in derselben Menge enthalten ist, wie in dem nadelförmigen, aus jenem leichter entweicht, als aus dem letzteren. Doch findet ein Verwittern beim Liegen an der Luft, wie es scheint; nicht leicht statt. Nach mehrtägigem Liegen gaben $0.5338 \mathrm{~g}$ des blätterigen Salzes noch immer $0 \cdot 1829 \mathrm{~g}$ Knpferoxyd, entsprechend $27 \cdot 33 \%$ Kupfer (fiir $\left[\mathrm{C}_{2} \mathrm{H}_{4} \mathrm{NO}_{2}\right]_{2} \mathrm{Cu}+\mathrm{H}_{2} \mathrm{O}$ berechnet: $27 \cdot 57 \%$ ). 
Dass ans diesem blätterigen Salz das Krystallwasser leichter. entweicht als aus dem gewöhnlichen Salz, ergab ein Versuch, bei dem beide Salze nebeneinander in demselben Trockenschrank auf $105-110^{\circ}$ erhitzt wurden. Während das nadelförmige Salz erst $4 \cdot 95 \%$ Wasser abgegeben hatte, war bei dem blätterigen Salz in der gleichen Zeit Gewichtsconstanz eingetreten unter einem Verlust von $7 \cdot 83 \%$ Wasser (berechnet: $7 \cdot 86 \%$ ). Möglicherweise ist also das Glycocollkupfer dimorph; man könnte hier allerdings auch an Isomerieverhältnisse denken, doch wollen wir uns der Erörterung dariiber hier enthalten.

\section{Orthotolylglycocollcalcium.}

$$
\left(\mathrm{C}_{9} \mathrm{H}_{10} \mathrm{NO}_{2}\right)_{2} \mathrm{Ca}+3 \mathrm{H}_{2} \mathrm{O} \text {. }
$$

In einer kurzen Mittheilung ïber die Gewinnung von Indol aus Phenylglycocoll ${ }^{1}$ haben wir eine Calciumverbindung dieses Glycocolls beschrieben, welche uns damals als Ausgangsproduct diente. Da bis dahin über derartige Calciumverbindungen von aromatisch substituirten Glycocollen keine Angaben vorlagen, so haben wir auch von dem $\boldsymbol{o}$-Tolylglycocoll und dem $\alpha$-Naphtylglycocoll (s. u.) die Calciumsalze hergestellt. Der dabei eingehaltene Vorgang war ganz derselbe, wie wir ihn seinerzeit beschrieben haben.

Das o-Tolylglycocollcalcium stellt, ausWasser umkrystallisirt, bis zolllange breite Nadeln von starkem Glanze dar, welche in Wasser ziemlich leicht, in verdinntem Weingeist schwerer, in absolntem Alkohol gar nicht löslich sind.

Eine Bestimmung des Krystallwassers war auch hier nicht ausführbar, da, sowie beim Phenylglycocollealcium, bei höherer Temperatur Zersetzung eintrat, das Salz iiber Schwefelsäure anderseits nichts abgab. Die Analysen wurden desshalb mit lufttrockener Substanz ausgetührt, wobei sich ergab, dass das Salz drei Molektile Wasser enthält. Die erhaltenen Zahlen waren folgende:

I. $0 \cdot 3297 g$ gaben $0 \cdot 6133 g$ Kohlensäure und $0 \cdot 1964 g$ Wasser. II. $0.3461 \mathrm{~g}$ gaben $21 \mathrm{~cm}^{3}$ Stickstoff bei $23 \cdot 5^{\circ}$ C. und $748 \mathrm{~mm}$ Barometerstand.

1 Monatshefte füil Chemie, X, 250.

Chemie-Heit NY. 7 u. 8 . 
III. $0.5767 \mathrm{~g}$ gaben $0.0784 \mathrm{~g}$ Calciumoxyd. IV. $0 \cdot 5346 g$ gaben $0.0722 g$ Calciumoxyd.
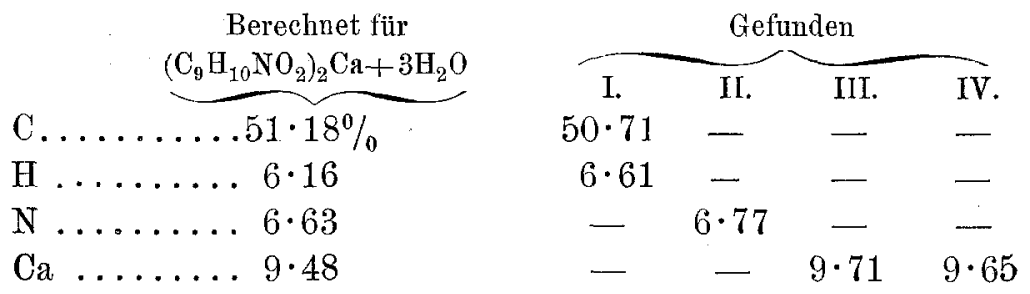

Bei der trockenen Destillation mit Calciumformiat entsteht neben basischen Producten ein Körper, der in analoger Weise, wie wir es beim Indol beschrieben haben, aus dem Gemenge isolirt und in das Pikrat übergefuhrt wurde. Dieses glich ganz dem Indolpikrat und lieferte bei der Destillation mit wässerigem Ammoniak einen dem Indol im Aussehen und Verhalten völlig gleichenden Körper in farblosen Blättern. Wir zweifeln nicht, in diesem Product das bereits von Ras chen ${ }^{1}$ in geringer Menge erhaltene $o$-Tolindol (B. 1. Methylindol) in Händen gehabt zu haben. Die Ausbente war bei diesem Indol jedoch nicht reich genug, um zu einer Darstellung in grösserem Masse anzuregen.

\section{IV. $\alpha$-Naphtylglycocoll.}

Schon in unserer vor zwei Jahren erschienenen Abhandlung: haben wir mitgetheilt, dass auch das $\alpha$-Naphtylglycocoll, welches damals noch nicht bekannt war, eine Calciumverbindung liefert. ${ }^{2}$ Wir hatten damals das $\alpha$-Naphtylglycocoll in Fortsetzung unserer Versuche der Darstellung substituirter Glycocolle unter Mitwirkung von Natriumcarbonat ${ }^{3}$ erhalten, indem wir $\alpha$-Naphtylaminchlorhydrat mit Sodalösung zerlegten und mit einem Überschuss von Soda und Chloressigsäure kochten. Wir können uns ersparen, Weiteres ubber diesen Körper hier mitzutheilen, da seither von drei Seiten darüber berichtet worden ist. ${ }^{4}$ Er zeigte die von den citirten Autoren angegebenen Eigenschaften, der Schmelzpunkt

1 Ann. 239, S. 228

2 Monatshefte für Chemie, X, 251.

3 Ebenda, IX, 727.

4 S. C. A. Bischoff und O. Nastvogel, Berichte XXII, 1808, O. Jolles, ebenda, 2372 und O. Forte, ebenda, Ref. 731. 
lag bei $192^{\circ}$ C., die Analysen ergaben 71.79 und $71.36 \%$ Kohlenstoff (berechnet: $71 \cdot 64$ ), $5 \cdot 72$ und $5 \cdot 66^{\%}$ Wasserstoff (berechnet: $5 \cdot 47$ ) und $7 \cdot 17 \%$ Stickstoff (berechnet: $6 \cdot 97$ ).

Das Calciumsalz wurde erhalten durch Lösen von Naphtylglycocoll in wenig Ammoniak und Fällen mit Chlorcalcium in der Hitze. Der Niederschlag wurde abgesangt, mit Wasser gewaschen und aus heissem Wasser oder verdünntem Weingeist umkrystallisirt, wobei das Salz in der Form farbloser Büschel von breiten Nadeln erhalten wurde. Auch hier war eine besondere Bestimmung des Krystallwassers nicht durchführbar. Die Analysen der Iufttrockenen Substanz ergaben:

I. $0 \cdot 2427 \mathrm{~g}$ gaben $0.5190 \mathrm{~g}$ Kohlensäure und $0.1203 \mathrm{~g}$ Wasser.

II. $0 \cdot 3130 \mathrm{~g}$ gaben $0 \cdot 0359 \mathrm{~g}$ Calciumoxyd.

III. $0 \cdot 3407 g$ gaben $0 \cdot 0396 g$ Calciumoxyd. IV. $0 \cdot 3533 g$ gaben $0 \cdot 0403 g$ Calciumoxyd.

Berechnet für

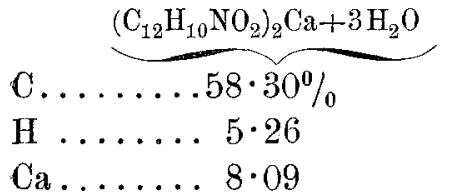

Gefunden

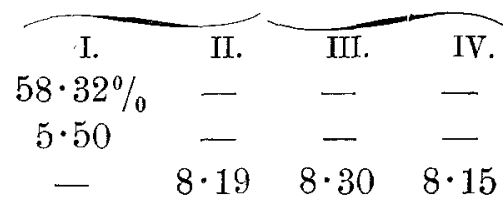

Das K upfersalz des $\alpha \cdot$ N a phtylglyeocolls wurde durch Fällen einer alkoholischen Lösung des Glycocolls mit wässerigem Kupferacetat in Form eines unscheinbaren dunkelbraunen Pulvers gewonnen, welches aus mikroskopischen Blättchen und kugeligen Krystallaggregaten bestand. Die lufttrockene Substanz ergab bei der Analyse folgende Werthe:

I. $0.3268 g$ gaben $0.1345 g$ Wasser, $0.7417 g$ Kohlensäure und $0 \cdot 0553 g$ Kupferoxyd.

II. $0.2572 \mathrm{~g}$ gaben $13.8 \mathrm{~cm}^{3}$ Stickstoff bei $11 \cdot 1^{\circ}$ C. und $742 \cdot 5 \mathrm{~mm}$ Barometerstand.

III. $0 \cdot 4191 g$ gaben $0 \cdot 0708 g$ Kupferoxyd.

\begin{tabular}{|c|c|c|c|}
\hline \multirow{2}{*}{$\begin{array}{r}\begin{array}{r}\text { Berechnet für } \\
\left(\mathrm{C}_{12} \mathrm{H}_{10} \mathrm{NO}_{2}\right)_{2} \mathrm{Cu}\end{array} \\
\end{array}$} & \multicolumn{3}{|c|}{ Gefunden } \\
\hline & I. & II. & III. \\
\hline C....62.18\% & $61 \cdot 90$ & - & - \\
\hline $\mathrm{H} \ldots 4 \cdot 32$ & $4 \cdot 57$ & - & - \\
\hline$N \ldots 6 \cdot 04$ & - & $6 \cdot 25$ & $\ldots$ \\
\hline $\mathrm{Cu} \ldots 13 \cdot 64$ & $13 \cdot 50$ & - & $13 \cdot 48$ \\
\hline
\end{tabular}


Bei der trockenen Destillation des $\alpha$-Naphtylglycincalciums mit Caleiumformiat und Behandeln des Destillates in der schon beschriebenen Weise wurde ein in schönen farblosen Blättchen krystallisirender Körper erhalten, welcher im Allgemeinen sehr ähnlich dem von Schli eper ${ }^{1}$ beschriebenen $\alpha-\mathrm{N}$ aph tindol "ist, sich jedoch durch das Ausbleiben der Reaction mit Wasserstoffsuperoxyd, sowie durch seinen Schmelzpunkt davon unterschied; der letztere lag bei $163^{\circ}$ und es gelang trotz wiederholten Umkrystallisirens aus verdünntem Weingeist, sowie durch Sublimiren nicht, denselben zu erhöhen.

\section{Phenylgly cin-p-Carbonsäure.}

$$
\mathrm{C}_{9} \mathrm{H}_{9} \mathrm{NO}_{4} \text {. }
$$

Analog der Darstellung der Phenylglycin-o.Carbonsäure konnte die entsprechende Paraverbindung erhalten werden, indem $25 g p$-Amidobenzoësäure, $20 \cdot 6 g$ Chloressigsäure, $32 \cdot \delta g$ kohlensaures Natrium in $1 l$ Wasser mehrere Stunden hindurch gekocht wurden. Die erkaltete Lösung liess beim Ansäuern reichliche Mengen $(30.2 g)$ eines sandigen, gelben Pulvers fallen, welches unter Anwendung von viel Wasser umkrystallisirt und als gelblich gefärbtes Krystallmehl erhalten werden konnte. Dasselbe ist in Wasser schwer, in Alkohol leichter löslich und schmilzt unter Zersetzung bei $219-221^{\circ} \mathrm{C}$.

Die Analyse der bei $105-110^{\circ}$ getrockneten Substanz ergab folgende Zahlen:

I. $0 \cdot 3244 \mathrm{~g}$ gaben $0 \cdot 1383 \mathrm{~g}$ Wasser und $0 \cdot 6633 \mathrm{~g}$ Kohlensäure.

II. $0 \cdot 3937 \mathrm{~g}$ gaben $25 \mathrm{~cm}^{3}$ Stickstoff bei $9^{\circ} \mathrm{C}$. und $744.4 \mathrm{~mm}$ Barometerstand.

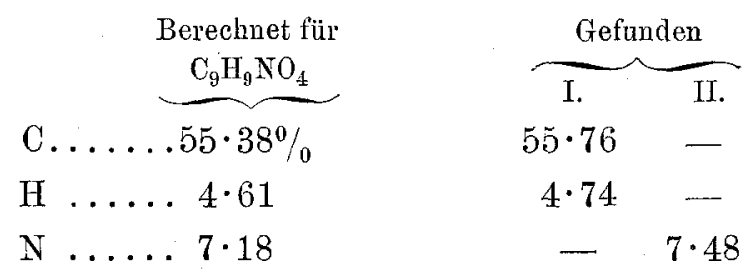

1 Ann. 239, S. 235.

2 Schlieper fand bei seinem $\alpha$-Naphtindol $174-175^{\circ}$. 
Baryumsalz $\mathrm{C}_{6} \mathrm{H}_{7} \mathrm{NO}_{4} \mathrm{Ba}+4 \mathrm{H}_{2} \mathrm{O}$. Durch Auflösen der Säure in der nöthigen Menge Ammoniakflüssigkeit und Fällen mit Chlorbaryum, Abfiltriren des Niederschlages, Waschen desselben mit Wasser, Aufösen in heissem Wasser, Fällen der erkalteten Lösung mit etwas Alkohol und neuerliches Auswaschen mit Wasser wurde dieses Salz in der Form eines weissen krystallinischen Pulvers erhalten.

Das Salz gab bei der Analyse folgende Zahlen:

I. $0 \cdot 3564 g$ gaben lufttrocken $0 \cdot 2045 g$ schwefelsauren Baryt.

II. $0.3157 \mathrm{~g}$ der lufttrockenen Substanz verloren bei 100 bis $110^{\circ}$ C. $0.0556 \mathrm{~g}$ Wasser und gaben $0.1534 \mathrm{~g}$ kohlensauren Baryt.

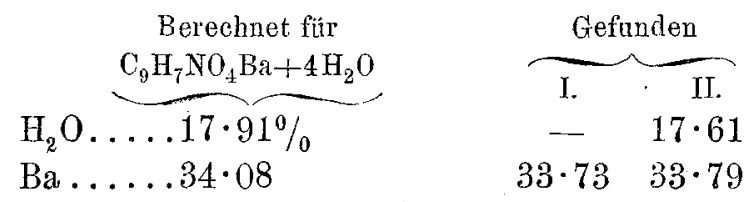

Das Calciu msalz der Phenylglycin-p-Carbonsäure, $\mathrm{C}_{9} \mathrm{H}_{7} \mathrm{NO}_{4} \mathrm{Ca}+3 \mathrm{H}_{2} \mathrm{O}$, wurde in ähnlicher Weise wie das Barynmsalz aus der Lösung des Ammoniaksalzes durch Zusatz von Chlorcalcium und Essigsäure gefällt, abgesaugt, gewaschen, in heissem Wasser gelöst, filtrirt, nach längerem Stehen mit Alkohol gefällt und mit Wasser gewaschen. Es stellt ein krystallinisches weisses Pulver dar, welches in Wasser ziemlich leicht löslich ist.

$0.5876 \mathrm{~g}$ des lufttrockenen Salzes verioren bei $100-110^{\circ} \mathrm{C}$. $0 \cdot 1092 g$ Wasser und gaben $0 \cdot 1112 g$ Calcinmoxyd.

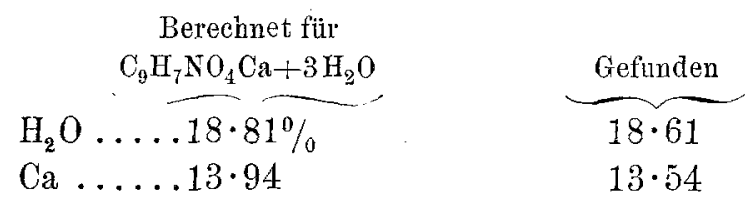

Das Kupfersalz; $\mathrm{C}_{9} \mathrm{H}_{7} \mathrm{NO}_{4} \mathrm{Cu}$, wurde durch Fällen der Ammoniakverbindung mit viel Kupfersulfat als dunkelgrüner, amorpher Niederschlag gewonnen, der gut gewaschen und im Exsiccator getrocknet wurde.

$0 \cdot 4725 g$ gaben $0 \cdot 1472 g$ Kupferoxyd. 
Berechnet für

$$
\mathrm{Cu} \ldots \underbrace{\mathrm{C}_{9} \mathrm{H}_{7} \mathrm{NO}_{4} \mathrm{Cu}}_{24 \cdot 67 \%} \quad \underbrace{\text { Gefunden }}_{24 \cdot 85 \%} \text {. }
$$

Ersetzt man die $p$-Amidobenzoësäure durch die Metaverbindung in der Absicht, eine Phenylglycin-m-Carbonsäure auf dem gleichen Wege zu erhalten, so entsteht nicht die erwartete Verbindung als Hauptproduct, sondern eine bei $210-212^{\circ}$ schmelzende Säure von der Formel $\mathrm{C}_{11} \mathrm{H}_{11} \mathrm{NO}_{6}$, welche wahrscheinlich als $m$-Carbonsäure der Phenyldiglycolamidsäure anzusehen ist. 